\title{
Development and Validation of an HPLC Method for Simultaneous Determination of Nine Active Components in 'Da-Chai-Hu-Tang'
}

\author{
Lingli Zheng, Deshi Dong* \\ The First Affiliated Hospital, Dalian Medical University, Dalian, China \\ E-mail: Zheng_1l2009@126.com \\ Received December 21, 2010; revised January 25, 2011; accepted February 2, 2011
}

\begin{abstract}
In this study, a simple, reliable and accurate method for the simultaneous separation and determination of naringin, hesperidin, neohesperidin, baicalin, wogonoside, baicalein, wogonin, emodin and chrysophanol in 'Da-Chai-Hu-Tang' was developed by reverse-phase high-performance liquid chromatography (RP-HPLC). The chromatographic separation was performed on an Agilent ZORBAX C18 column $(250 \mathrm{~mm} \times 4.6 \mathrm{~mm}$ i.d., $5.0 \mu \mathrm{m})$, and the mobile phase composed of methanol and water containing $1 \%(\mathrm{v} / \mathrm{v})$ acetic acid was used to elute the targets in a gradient elution mode. The flow rate and detection wavelength were set at 0.8 $\mathrm{ml} / \mathrm{min}$ and $280 \mathrm{~nm}$, respectively. All calibration curves of the nine components expressed good linearities ( $r 2 \geq 0.9992)$ within the tested ranges. The RSD values demonstrated the intra- and inter-day precisions were less than $2.89 \%$, and the recoveries of the investigated compounds were between $96.22 \%$ and $105.28 \%$. The proposed method is simple, precise, specific, sensitive, and successfully applied to determine the nine marker compounds in 'Da-Chai-Hu-Tang' for quality control.
\end{abstract}

Keywords: High-Performance Liquid Chromatography, ‘Da-Chai-Hu-Tang’, Traditional Chinese Medicine, Multiple Compounds Determination

\section{Introduction}

Traditional Chinese medicines (TCMs), especially in China, have played an important role in clinical therapy, and been widely used for the prevention and treatment various diseases because of its high effectiveness and low toxicity for thousands of years $[1,2]$.

Generally, herbal medicines are used in combinations to afford a formula composed of several single herbs. Combining the herbs together and boiled in solvent can make different preparations, and multiple constituents are usually responsible for the therapeutic effects by synergistic or antagonistic interactions. Each herb has its own bioactivities, but when many herbs are combined, there may be changes of active components, especially in their contents. Moreover, some TCMs have been widely administrated directly after boiling with water without any quality assessment in some areas of China, which may produce some side effects and influence the activities of herbal products because of different herbs from different regions with different contents of active com- pounds. That is why the quality of TCMs is very critical important for affording the efficiency and avoiding the toxicity. Thus, sensitive and reliable holistic analytical approach is necessary.

Mostly, single marker compound is analyzed to evaluate the quality of TCMs [3], which is simple but cannot totally demonstrate the quality of herbal prescriptions. Then, multiple components analysis (MCA) method has been developed, which can simultaneously evaluate many active compounds from different herbs and has been widely used for the quality control of TCMs [4-6].

In the process of component determination, analytical methods and technologies are essential. Up to date, two kinds of chromatographic techniques, high-performance liquid chromatography (HPLC) and HPLC-mass spectrometry (HPLC-MS), have been used more and more frequently for the quality control of various kinds of herbal medicines [7-9]. The former, has been universally used as a convenient and sensitive method because of its convenience, precision, cheapness, sensitivity and reproducibility [10]. The later, can screen the chemical constitu- 
ents high-throughput in TCMs, especially those trace components which are difficult for analysis by conventional methods. Hence, HPLC-MS is a powerful tool for its high level of sensitivity and selectivity, but the expensive running cost violates its application in routine analysis. Thus, in this paper, HPLC method was established to achieve quality control.

The Chinese formular 'Da-Chai-Hu-Tang' (DCHT), is a botanical drug and composed of Radix bupleuri, Fructus aurantii immaturus, Rhizoma zingiberis recens, Radix scutellariae, Radix paeoniae alba, Rhizoma pinelli and Fructus jujubae. Because of its therapeutic effectiveness and few side effects, DCHT has been widely used to treat acute cholecystitis, cholelithiasis, pancreatitis and appendicitis in China [11]. By now, pharmacological research has demonstrated that it also shows a good effect in inhibition atherosclerosis and fatty liver $[12,13]$.

In this decoction, there are several compounds with significant pharmacological effects, such as flavonoids including naringin and hesperidin from F. aurantii immaturus [14-18], baicalin and wogonoside from $R$. scutellariae [19-24], and anthraquinones including emodin and chrysophanol from $R$. paeoniae alba [25-29], etc. Thus, selection of these marker compounds for totally quality research of DHCT is critical important. But there have no papers reported for simultaneous determination of the nine marker compounds in DHCT for quality control through a literature search as far as we known.

The aim of the present paper was to establish a simple, efficient and sensitive method for simultaneous analysis of nine marker compounds including naringin, hesperidin, neohesperidin, baicalin, wogonoside, baicalein, wogonin, emodin and chrysophanol (shown in Figure 1) in DHCT for quality control by HPLC.

\section{Experimental}

\subsection{Materials and Reagents}

Nine standard compounds of naringin, hesperidin, neohesperidin, baicalin, wogonoside, baicalein, wogonin, emodin and chrysophanol were purchased from the National Institute for Control of Pharmaceuticals and Biological Products (Beijing, China). Medicinal plants, $R a-$ dix bupleuri, Fructus aurantii immaturus, Rhizoma zingiberis recens, Radix scutellariae, Radix paeoniae alba, Rhizoma pinelli, Fructus jujubae and Radix et Rhizoma Rhei were purchased from a local drug store (Dalian, China) and identified by Dr. Yun-Peng Diao (Dalian Medical University, Dalian, China). Voucher specimens were deposited in College of Pharmacy, Dalian Medical University (Dalian, China). Methanol was HPLC grade (TEDIA, USA), and water for HPLC analysis was prepared using a Millipore (Millipore, USA). Acetic acid and other reagents were analytical grade purchased from ShenLian Chemical Factory (Shenyang, China). All the solvents and solutions were filtered through a Millipore filter $(0.45 \mu \mathrm{m})$ before use.

\subsection{Standard Solution Preparation}

A mixed stock standard solution containing naringin, hesperidin, neohesperidin, baicalin, wogonoside, baicalein, wogonin, emodin and chrysophanol was prepared by<smiles>[R]c1cc(O)c2c(c1)OC(c1ccc(O)cc1)CC2=O</smiles>

(1) $R=2-O-\alpha$-L-rhamnosyl-D-glucoside<smiles>COc1ccc([C@H]2CC(=O)c3c(O)cc(O[C@@H]4O[C@H](CO)[C@@H](O)[C@H](O)[C@H]4OC4O[C@H](C)[C@@H](O)[C@H](O)[C@H]4O)cc3O2)cc1O</smiles>

(3)<smiles>COc1ccc([C@@H]2CC(=O)c3c(O)cc(O[C@@H]4O[C@H](CO[C@H]5C[C@H](C)[C@@H](O)[C@H](O)[C@H]5O)[C@@H](O)[C@H](O)[C@H]4O)cc3O2)cc1O</smiles>

(2)<smiles>[R20]c1c([R])c(O)c2c(=O)cc(-c3ccccc3)oc2c1[R]</smiles><smiles>[R]c1cc(O)c2c(c1)C(=O)c1cc([R])cc(O)c1C2=O</smiles>
(4) $\mathrm{R}_{1}=\mathrm{H} \quad \mathrm{R}_{2}=$ glcA $\quad \mathrm{R}_{3}=\mathrm{OH}$
(5) $\mathrm{R}_{1}=\mathrm{OCH}_{3} \quad \mathrm{R}_{2}=$ glcA $\quad \mathrm{R}_{3}=\mathrm{H}$
(6) $\mathrm{R}_{1}=\mathrm{R}_{2} \quad \mathrm{R}_{3}=\mathrm{OH}$
(7) $\mathrm{R}_{1}=\mathrm{OCH}_{3} \quad \mathrm{R}_{2}=\mathrm{R}_{3}=\mathrm{H}$

(8) $\mathrm{R}_{1}=\mathrm{OH} \quad \mathrm{R}_{2}=\mathrm{CH}_{3}$

(9) $\mathrm{R}_{1}=\mathrm{H} \quad \mathrm{R}_{2}=\mathrm{COOH}$

Figure 1. The chemical structures of the nine components: (1) naringin; (2) hesperidin; (3) neohesperidin; (4) baicalin; (5) wogonoside; (6) baicalein; (7) wogonin; (8) emodin and (9) chrysophanol. 
accurately weighing appropriate amounts of the nine reference compounds and dissolving in methanol. All the standard stock and working solutions were prepared in dark brown calibrated flasks and stored at $4^{\circ} \mathrm{C}$.

\subsection{Preparation of Sample Solutions and Negative Control Solutions}

Ten medical plants were triturated into powders in the particle size of 40-60 mesh, and then weighed according to DCHT formula and blended. The mixed powder $(0.70$ g) was extracted by $20 \mathrm{ml}$ methanol for $20 \mathrm{~min}$ in an ultrasonic bath. In order to keep the repeatability of the extraction procedure, lost volume of methanol was compensated after extraction. After filtration, $2 \mathrm{ml}$ filtrate was transferred into a $10 \mathrm{ml}$ volumetric flask with $\mathrm{MeOH}$ and $10 \mu \mathrm{l}$ of the resultant solution was injected into the LC system for analysis after through a $0.45 \mu \mathrm{m}$ Millipore filter.

According to the prescription and preparation protocol of DCHT formula, three kinds of negative control samples in which the formula without F. aurantii Immaturus, $R$. scutellariae, or $R$. et Rhizoma Rhei, respectively, were prepared to validate the specificity of the method. The negative samples were prepared according to the method mentioned above.

\subsection{Apparatus and Chromatographic Conditions}

Chromatography was performed with an Agilent Technologies 1200 series HPLC system consisting of a quaternary delivery system, an auto-sampler and a DAD detector. All the separations were carried out on a ZORBAX SB C18 column (250 mm $\times 4.6 \mathrm{~mm}$ I.D., 5 $\mu \mathrm{m})$. The mobile phase consisted of methanol (A) and water containing $1 \%$ acetic acid (B) at a flow rate of 0.8 $\mathrm{ml} / \mathrm{min}$ with a gradient elution mode was carried out as follows: 0-20 min, linear gradient from $15 \%$ A to $35 \% \mathrm{~A}$; 20-40 min, the mobile phase was held on 35\% A; 40-60 min, linear gradient to $50 \% \mathrm{~A} ; 60-110 \mathrm{~min}$, the linear gradient to $80 \% \mathrm{~A} ; 110-120 \mathrm{~min}$, the linear gradient to $95 \%$ A. Each run was followed by equilibration time of 15 min. Ultraviolet (UV) spectra were monitored at 280 $\mathrm{nm}$. All the data were collected and analyzed with Chemstation software.

\section{Results and Discussions}

\subsection{Optimization of Chromatographic Conditions}

To develop an accurate, valid and optimal chromatogram, some HPLC analytical parameters including separation column, mobile phase and its elution mode were all investigated in this study. Four kinds of reversed-phase columns, Lichrosorb C18 column $(150 \mathrm{~mm} \times 4.6 \mathrm{~mm}$ I.D., $5 \mu \mathrm{m})$, Johnsson ODS2 C18 column $(250 \mathrm{~mm} \times 4.6$ mm I.D., $5 \mu \mathrm{m})$, Agilent XDB C18 column $(150 \mathrm{~mm} \times$ $4.6 \mathrm{~mm}$ I.D., $5 \mu \mathrm{m})$ and Agilent ZORBAX C18 column (250 $\mathrm{mm} \times 4.6 \mathrm{~mm}$ I.D., $5 \mu \mathrm{m}$ ) were tested under different elution modes of using methanol-water or acetonitrile-water containing different concentrations of acetic acid as the mobile phase (listed in Table 1). After a serial of experiments, we found that the separation was performed on an Agilent ZORBAX C18 column (250 mm $\times$ $4.6 \mathrm{~mm}$ I.D., $5 \mu \mathrm{m}$ ) using the solvent system composed of methanol (A)-water containing 1\% acetic acid (B) as the mobile phase with gradient elution mode as follows: 0-20 min, linear gradient from 15\% A to 35\% A; 20-40 min, the mobile phase was held on 35\% A; 40-60 min, linear gradient to $50 \% \mathrm{~A}$; $60-110 \mathrm{~min}$, the linear gradient to $80 \% \mathrm{~A} ; 110-120 \mathrm{~min}$, the linear gradient to $95 \% \mathrm{~A}$. The flow rate was $0.8 \mathrm{ml} / \mathrm{min}$. The detection wavelength was set at $280 \mathrm{~nm}$ on the basis of the UV spectra with three dimension chromatograms of DAD detection, where all the compounds could be detected and had adequate adsorption. Selectivity was assessed by comparing chromatograms obtained from the blank sample and from the corresponding spiked sample. Typical chromatograms are shown in Figure 2, in which chromatograms of $\mathrm{A}, \mathrm{B}$ and $\mathrm{C}$ correspond to blank mobile phase, mixed standards, DCHT, and the peaks $1,2,3,4$, 5, 6, 7, 8 and 9 represent naringin, hesperidin, neohesperidin, baicalin, wogonoside, baicalein, wogonin, emodin and chrysophanol, respectively.

\subsection{Optimization Sample Extraction Protocol}

The extraction conditions, for example extraction solvent, method and time, can easily influence the efficiency of the extraction. As a result, it is necessary to estimate and optimize the factors affecting extraction recovery. Two methods, boiling and ultrasonic are often used to extract the targets from matrix. The disadvantages of the boiling procedure are the loss of the compounds due to ionization, hydrolysis and oxidation during extraction, the consumption of a large amount of solvent, low extraction efficiency, and time-consuming. These shortcomings have led to the consideration of ultrasound-assisted extraction (UAE) method, which has been widely used in quality control of TCMs. In UAE process, extraction solvent, sample-solvent ratio and extraction time are critical important for high extraction efficiency.

Methanol is often used as the extraction solvent because of its high efficiency and directly application for 
Table 1. The tried column and mobile phase in optimization of HPLC conditions.

\begin{tabular}{|c|c|c|}
\hline Column & Solvent system & Elution mode \\
\hline $\begin{array}{l}\text { Lichrosorb } \mathrm{C}_{18}(4.6 \mathrm{~mm} \times 150 \mathrm{~mm} \text { I.D., } 5 \mu \mathrm{m}) \\
\text { (Zhonghuida, Dalian, China) }\end{array}$ & $\begin{array}{l}\text { Acetonitrile (A) } \\
\text { and water (B) }\end{array}$ & 0 20 min, 15\% A; 20 30 min, 15\% 40\% A; 30 60 min, 40\% A \\
\hline $\begin{array}{l}\text { ODS2 } \mathrm{C}_{18}(4.6 \mathrm{~mm} \times 250 \mathrm{~mm} \text { I.D., } 5 \mu \mathrm{m}) \\
\text { (Johnsson, Dalian, China) }\end{array}$ & $\begin{array}{l}\text { Acetonitrile (A) } \\
\text { and water (B) }\end{array}$ & 0 30 min, 10 40\% A; 30 60 min, 40\% A \\
\hline $\begin{array}{l}\text { ODS2 } \mathrm{C}_{18}(4.6 \mathrm{~mm} \times 250 \mathrm{~mm} \text { I.D., } 5 \mu \mathrm{m}) \\
\text { (Johnsson, Dalian, China) }\end{array}$ & $\begin{array}{l}\text { Methanol (A) } \\
\text { And water (B) }\end{array}$ & $\begin{array}{l}0 \sim 10 \min , 10 \% \text { A; 10 40 min, 10 40\% A; 40 80 min, } 40 \% \text { A; } \\
80 \sim 120 \mathrm{~min}, 40 \sim 60 \% \mathrm{~A}\end{array}$ \\
\hline $\begin{array}{l}\text { XDB } \mathrm{C}_{18}(4.6 \mathrm{~mm} \times 150 \mathrm{~mm} \text { I.D., } 5 \mu \mathrm{m}) \\
\text { (Agilent, USA) }\end{array}$ & $\begin{array}{l}\text { Methanol (A) } \\
\text { and water (B) }\end{array}$ & $\begin{array}{l}0 \sim 15 \min , 10 \sim 30 \% \mathrm{~A} ; 15 \sim 30 \mathrm{~min}, 30 \sim 40 \% \mathrm{~A} ; 30 \sim 40 \mathrm{~min}, 40 \% \\
\mathrm{~A} ; 40 \sim 60 \mathrm{~min}, 40 \sim 60 \% \mathrm{~A} ; 60 \sim 80 \mathrm{~min}, 60 \sim 80 \% \mathrm{~A}\end{array}$ \\
\hline $\begin{array}{l}\mathrm{ZORBAX} \mathrm{C}_{18}(4.6 \mathrm{~mm} \times 250 \mathrm{~mm} \text { I.D., } 5 \mu \mathrm{m}) \\
\text { (Agilent, USA) }\end{array}$ & $\begin{array}{l}\text { Methanol (A) } \\
\text { and } 1 \% \text { acetic acid water (B) }\end{array}$ & $\begin{array}{l}0 \sim 15 \mathrm{~min}, 20 \sim 40 \% \mathrm{~A} ; 15 \sim 35 \mathrm{~min}, 40 \% \mathrm{~A} ; 35 \sim 40 \mathrm{~min}, 40 \sim 45 \% \\
\mathrm{~A} ; 40 \sim 60 \mathrm{~min}, 45 \sim 60 \% \mathrm{~A} ; 60 \sim 100 \mathrm{~min}, 60 \sim 95 \% \mathrm{~A}\end{array}$ \\
\hline $\begin{array}{l}\mathrm{ZORBAX} \mathrm{C}_{18}(4.6 \mathrm{~mm} \times 250 \mathrm{~mm} \text { I.D., } 5 \mu \mathrm{m}) \\
\text { (Agilent, USA) }\end{array}$ & $\begin{array}{l}\text { Methanol (A) } \\
\text { and } 1 \% \text { acetic acid water }(\mathrm{B})\end{array}$ & $\begin{array}{l}0 \sim 10 \min , 22 \sim 35 \% \text { A; 10 30 min, 35 38\% A; 30 40 min, } \\
\text { 38 45\% A; 40 60 min, 45 60\% A; 60 100 min, 60 95\% A }\end{array}$ \\
\hline $\begin{array}{l}\mathrm{ZORBAX} \mathrm{C}_{18}(4.6 \mathrm{~mm} \times 250 \mathrm{~mm} \text { I.D., } 5 \mu \mathrm{m}) \\
\text { (Agilent, USA) }\end{array}$ & $\begin{array}{l}\text { Methanol (A) } \\
\text { and } 1 \% \text { acetic acid water (B) }\end{array}$ & $\begin{array}{l}\text { 0 20 min, 15 35\% A; 20 40 min, 35\% A; 40 60 min, 35 50\% } \\
\text { A; 60 110 min, 50 80\% A; 110 120 min, 80 95\% A }\end{array}$ \\
\hline
\end{tabular}

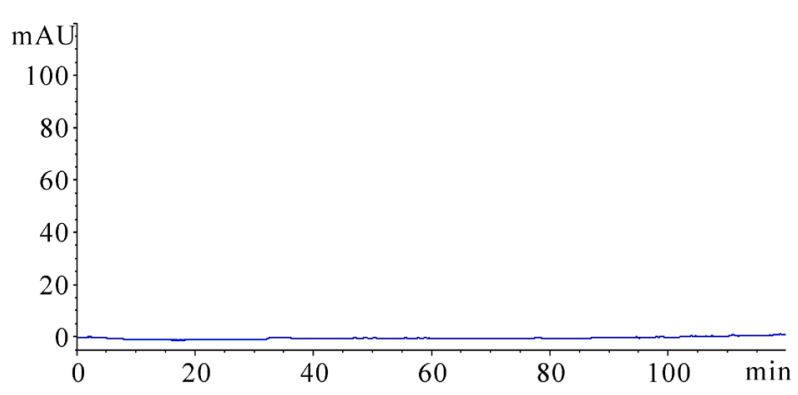

(a)

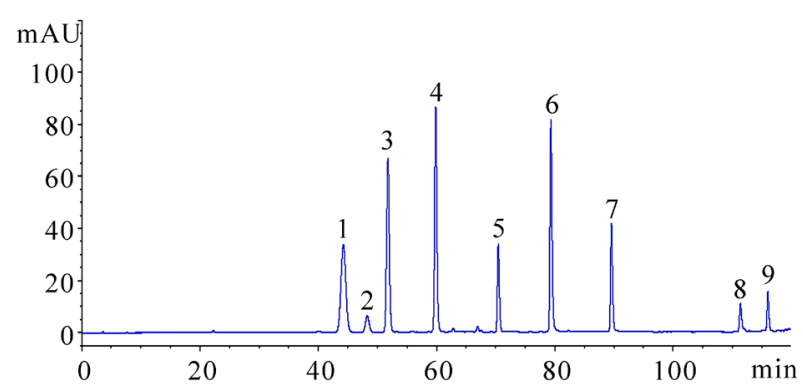

(b)

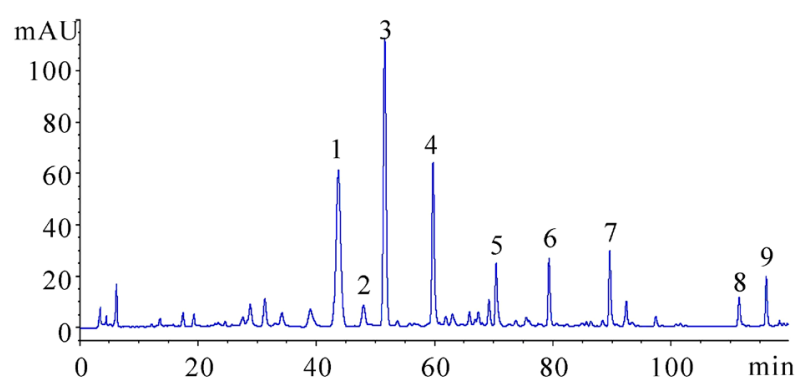

(c)

Figure 2. Representative HPLC chromatograms of: (a) mobile phase; (b) mixed standard solutions; (c) DCHT sample.
HPLC analysis without any more preparation. In the present paper, pure and aqueous methanol (20\%, 40\%, $60 \%$ and $80 \%$ ) were tried and examined as theextraction solvent for DCHT by UAE for $30 \mathrm{~min}$. The results shown in Figure 3(a) showed that the extraction rates of all targets were gradually increased along with the increase of methanol concentrations, and pure methanol was selected as the extraction solvent. Second, three levels of the use of methanol $(10,20$ and $30 \mathrm{ml})$ were investigated, and the results are shown in Figure 3(b). It was evident that $20 \mathrm{ml}$ methanol was the best for the extraction. Furthermore, the extract time, including 10, 20, 30 and 45 min were also optimized and the result shown in Figure 3(c) indicated that the extraction time controlled at 20 min was enough. In the end, the suitable extraction conditions were as follows: the samples were extracted by UAE using $20 \mathrm{ml}$ methanol as the extraction solvent, and the process was lasted for $20 \mathrm{~min}$.

\subsection{Specificity of the Method}

In order to investigate the specificity of the method, different negative control samples of DHCT were prepared and analyzed by HPLC, and the chromatograms are shown in Figure 4. It was obvious that there were no interferences for determination of the nine compounds by comparing the retention times with the standards. Furthermore, the purities of the investigated peaks were all confirmed to be pure through DAD purity studies.

\subsection{Calibration Curves, the Limit of Detection (LOD) and Quantification (LOQ)}

The external standard method was used to obtain regression 


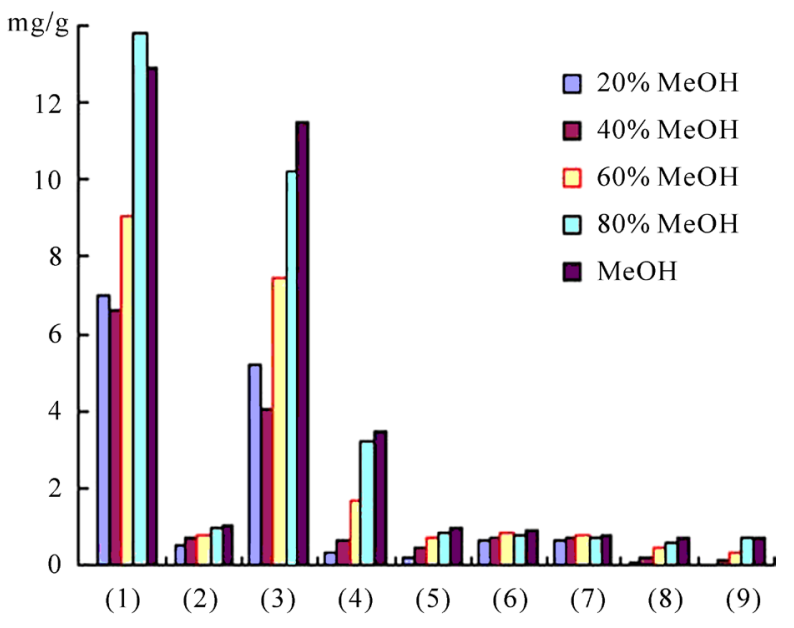

(a)

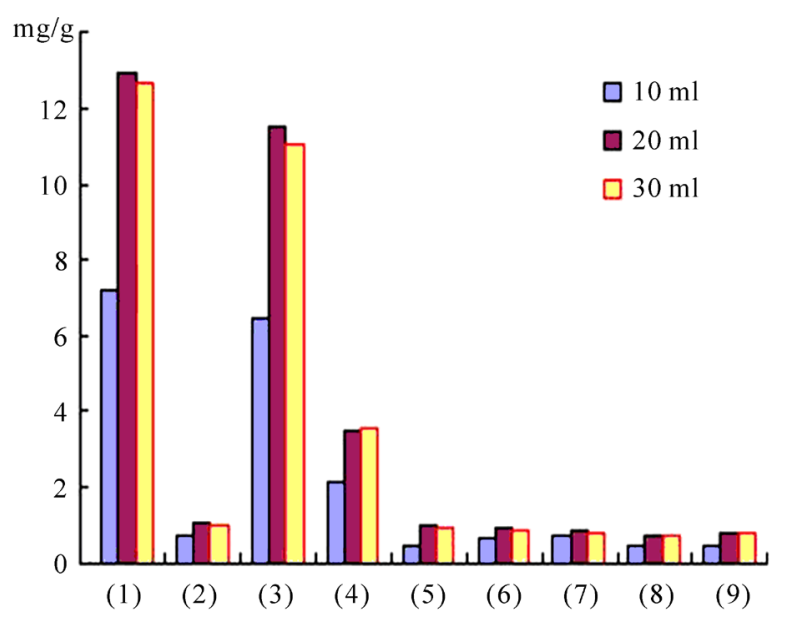

(b)

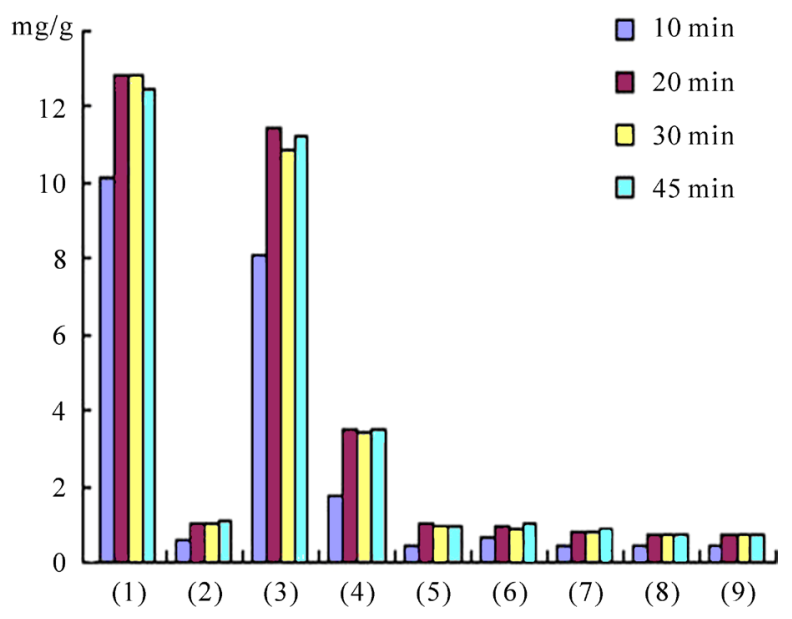

(c)

Figure 3. Efficiencies of the extraction for the nine compounds in DCHT using different: (a) extraction solvent; (b) the use of methanol; (c) extraction time.



(a)

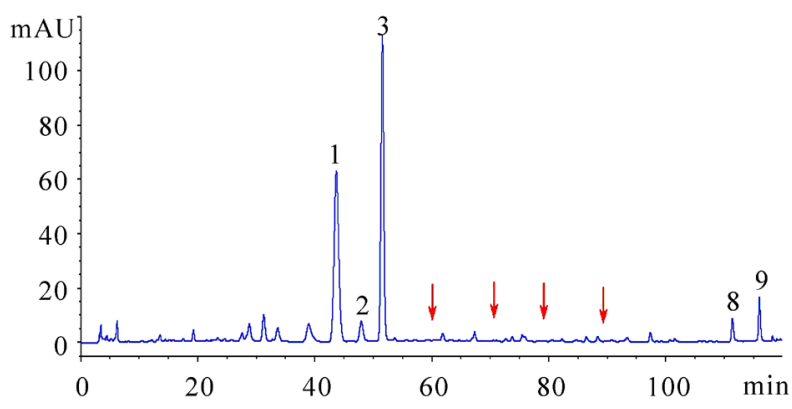

(b)

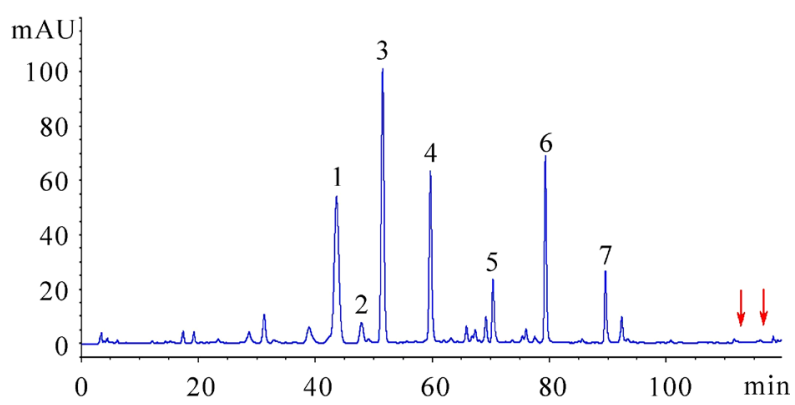

(c)

Figure 4. Representative HPLC chromatograms of: (a) the negative sample without $F$. aurantii immaturus; (b) the negative sample without $R$. scutellariae; (c) the negative sample without R.et Rhizoma Rhei.

equations. The calculated results are shown in Table 2. In the regression equation $y=a x+b, y$ refers to the peak area, $x$ is the concentration of the standard compounds $(\mu \mathrm{g} / \mathrm{ml})$, while $\mathrm{a}$ is the slope rate of the line and $\mathrm{b}$ is the intercept of the straight line with y-axis. All the standard compounds showed good linearity ( $\mathrm{r} 2 \geq 0.9992)$ in the tested concentration ranges. The limit of detection (LOD) and quantification (LOQ) were also measured. The standard solution was diluted with MEOH to the appropriate concentrations. The detection limit was defined as the lowest concentration level resulting in a peak area of three times the baseline noise. LOD was in the range of $0.07-0.30 \mu \mathrm{g} / \mathrm{ml}$. The LOQ was obtained as amount to give a signal-to-noise ratio $(\mathrm{S} / \mathrm{N})$ of 10 in the range of 0.35-0.87 $\mu \mathrm{g} / \mathrm{ml}$ (listed in Table 2). 
Table 2. Regression data, linear range and the LOD and LOQ of the developed method.

\begin{tabular}{lccccc}
\hline \multicolumn{1}{c}{ Analytes } & Calibration curve $^{\mathrm{a}}$ & $r^{2}$ & Linear range $(\mu \mathrm{g} / \mathrm{ml})$ & LOD $^{\mathrm{b}}(\mu \mathrm{g} / \mathrm{ml})$ & $\mathrm{LOQ}^{\mathrm{c}}(\mu \mathrm{g} / \mathrm{ml})$ \\
\hline Naringin & $y=17.40 x+20.12$ & 1.0000 & $1.53-49.00$ & 0.25 & 0.75 \\
Hesperidin & $y=21.75 x+12.72$ & 0.9999 & $2.70-43.20$ & 0.20 & 0.48 \\
Neohesperidin & $y=23.38 x+28.20$ & 0.9999 & $9.75-312.00$ & 0.08 & 0.40 \\
Baicalin & $y=41.80 x-80.41$ & 0.9998 & $6.25-200.00$ & 0.07 & 0.35 \\
Wogonoside & $y=45.22 x+62.24$ & 0.9998 & $3.25-120.00$ & 0.18 & 0.50 \\
Baicalein & $y=68.27 x-36.53$ & 0.9994 & $1.25-40.00$ & 0.30 & 0.55 \\
Wogonin & $y=64.31 x-105.86$ & 0.9996 & $3.12-50.00$ & 0.30 & 0.74 \\
Emodin & $y=25.72 x+30.86$ & 0.9993 & $4.00-64.00$ & 0.25 & 0.87 \\
Chrysophanol & $y=33.47 x+23.22$ & 0.9992 & $2.38-76.00$ & 0.28 & 0.74 \\
\hline
\end{tabular}

$y$ is the peak area in HPLC analysis monitored at $280 \mathrm{~nm}, x$ is the concentration of compound $(\mu \mathrm{g} / \mathrm{ml})$; ${ }^{\mathrm{b}} \mathrm{LOD}$ refers to the limit of detection, $S / N=3$; ${ }^{c} \mathrm{LOQ}$ refers to the limit of quantification, $S / N=10$.

\subsection{Assay Precision, Repeatability, Stability and Recovery}

The precision of the method was validated by both intraand inter-day precisions. The assays were carried out on the same mixed standard solutions at low, medium and high concentration levels during one day and one assay each day for three consecutive days, respectively. Relative standard deviation (RSD) of the mean content for each compound was calculated and ranged from $0.46 \%$ to $2.89 \%$ for intra- and inter-day precisions, which is shown in Table 3. The results indicated that the accuracy and precision of the proposed method were sufficient for determination of the nine compounds in the sample of DCHT.

The analysis repeatability of the nine components (Table 4) was determined by analysis of six samples which were prepared with the same preparation procedure and processed in parallel as described above. The RSD was calculated as a measurement for the repeatability of the method. The results indicated that the RSD values of each compound detected were all less than $1.97 \%$, which showed good reproducibility of the developed method.

For the stability test, a sample of DCHT was analyzed with the interval of $6 \mathrm{~h}(0,6,12$ and $18 \mathrm{~h})$ at room temperature, and the sample solution was found to be stable (RSD values of the mean content were lower than 2.15\%). The results are listed in Table 4.

The recovery assays were carried out by adding known contents of the standard samples to the known amounts of samples of DCHT and comparing the determined amount of these standards with the amount originally added. Table 4 shows these results of recovery tests. The mean recovery of the method was in the range of $96.22-105.28 \%$, with RSD of less than $2.33 \%$. Considering the results of the recovery assays, the method was thus acceptable.

Table 3. Intra- and inter-day variability of the 9 analytes.

\begin{tabular}{|c|c|c|c|c|c|}
\hline \multirow[b]{2}{*}{ Analytes } & \multirow{2}{*}{$\begin{array}{l}\text { Concentration } \\
(\mu \mathrm{g} / \mathrm{ml})\end{array}$} & \multicolumn{2}{|c|}{ Intra-day $(n=6)$} & \multicolumn{2}{|c|}{ Inter-day $(n=3)$} \\
\hline & & $\begin{array}{c}R S D^{a} \\
(\%)\end{array}$ & $\begin{array}{c}\text { Accuracy }^{b} \\
(\%)\end{array}$ & $R S D(\%)$ & $\begin{array}{c}\text { Accuracy } \\
(\%)\end{array}$ \\
\hline & 3.06 & 1.87 & 98.37 & 2.89 & 96.08 \\
\hline \multirow[t]{3}{*}{ Naringin } & 6.12 & 2.06 & 102.61 & 2.04 & 103.43 \\
\hline & 24.50 & 2.69 & 98.78 & 2.49 & 99.22 \\
\hline & 5.40 & 1.97 & 98.70 & 1.27 & 96.11 \\
\hline \multirow[t]{3}{*}{ Hesperidin } & 10.80 & 1.83 & 102.13 & 0.89 & 99.63 \\
\hline & 21.60 & 1.64 & 103.70 & 1.64 & 101.99 \\
\hline & 19.50 & 0.86 & 97.23 & 1.97 & 98.56 \\
\hline \multirow[t]{3}{*}{ Neohesperidin } & 78.00 & 1.94 & 102.32 & 2.46 & 98.96 \\
\hline & 312.00 & 2.06 & 100.98 & 1.48 & 96.51 \\
\hline & 12.50 & 2.29 & 101.20 & 1.83 & 101.52 \\
\hline \multirow[t]{3}{*}{ Baicalin } & 50.00 & 1.64 & 96.70 & 0.94 & 97.52 \\
\hline & 100.00 & 0.73 & 97.62 & 0.81 & 98.19 \\
\hline & 7.50 & 0.94 & 98.13 & 1.94 & 98.80 \\
\hline \multirow[t]{3}{*}{ Wogonoside } & 30.00 & 0.46 & 103.60 & 2.19 & 96.90 \\
\hline & 60.00 & 1.33 & 96.40 & 1.45 & 97.88 \\
\hline & 2.50 & 1.81 & 96.40 & 1.70 & 102.80 \\
\hline \multirow[t]{3}{*}{ Baicalein } & 10.00 & 1.90 & 98.50 & 1.54 & 98.10 \\
\hline & 20.00 & 2.05 & 103.85 & 0.96 & 96.20 \\
\hline & 6.25 & 1.05 & 98.08 & 1.57 & 97.12 \\
\hline \multirow[t]{3}{*}{ Wogonin } & 12.50 & 0.46 & 99.52 & 2.06 & 98.88 \\
\hline & 25.00 & 1.07 & 98.76 & 2.16 & 101.40 \\
\hline & 8.00 & 1.41 & 99.75 & 1.62 & 99.25 \\
\hline \multirow[t]{3}{*}{ Emodin } & 16.00 & 0.99 & 97.94 & 1.97 & 102.63 \\
\hline & 32.00 & 0.71 & 98.50 & 2.05 & 102.84 \\
\hline & 4.75 & 1.45 & 98.11 & 0.87 & 100.42 \\
\hline \multirow[t]{2}{*}{ Chrysophanol } & 9.50 & 1.70 & 101.16 & 1.67 & 98.74 \\
\hline & 38.00 & 1.11 & 99.05 & 1.56 & 98.66 \\
\hline
\end{tabular}


${ }^{\mathrm{a}} \mathrm{RSD}(\%)=(\mathrm{SD} / \mathrm{mean}) \times 100 ;{ }^{\mathrm{b}}$ accuracy $(\%)=($ mean of measured concen- $\quad$ tration/spiked concentration $) \times 100$.

Table 4. Repeatbility, stability and recovery results for the assay of the 9 analytes.

\begin{tabular}{|c|c|c|c|c|c|c|}
\hline \multirow{2}{*}{ Analyte } & \multicolumn{2}{|c|}{ Repeatability $(n=6)$} & \multicolumn{2}{|c|}{ Stability (18 h, $n=3$ ) } & \multicolumn{2}{|c|}{ Recovery $^{\mathrm{a}}(n=3)$} \\
\hline & Mean $(\mathrm{mg} / \mathrm{g})$ & $R S D(\%)$ & Mean $(\mathrm{mg} / \mathrm{g})$ & $R S D(\%)$ & Recovery (\%) & $R S D(\%)$ \\
\hline Naringin & 13.92 & 0.94 & 13.87 & 1.68 & 98.65 & 1.40 \\
\hline Hesperidin & 1.06 & 1.37 & 1.02 & 0.67 & 99.03 & 2.33 \\
\hline Neohesperidin & 11.51 & 0.48 & 11.46 & 1.27 & 96.22 & 1.00 \\
\hline Baicalin & 3.49 & 1.32 & 3.52 & 0.80 & 101.04 & 0.81 \\
\hline Wogonoside & 0.98 & 0.87 & 0.94 & 0.94 & 98.33 & 0.48 \\
\hline Baicalein & 0.92 & 0.94 & 0.95 & 0.73 & 105.28 & 0.92 \\
\hline Wogonin & 0.84 & 1.38 & 0.86 & 2.15 & 97.17 & 1.05 \\
\hline Emodin & 0.70 & 1.97 & 0.73 & 1.67 & 98.73 & 1.23 \\
\hline Chrysophanol & 0.78 & 0.86 & 0.77 & 1.38 & 97.54 & 1.44 \\
\hline
\end{tabular}

${ }^{\mathrm{a}}$ Recovery $(\%)=($ detected amount - original amount $) /$ spiked amount $\times 100$.

\section{Conclusions}

An HPLC method for simultaneous determination of nine active compounds including rhaponticin, naringin, hesperidin, neohesperidin, baicalin, wogonoside, baicalein, wogonin, emodin and chrysophanol in DHCT has not been reported. The presented method in addition to its novelty for determination of nine ingredients was sufficiently rapid, simple and sensitive as well as precise and accurate, and it was not interfered with other chemical constituents in DCHT. The linearity, accuracy, precision, LOD and LOQ, specificity-selectivity of the method and sample stability were all established. Although nine compounds were quantitated, there are many other components in DCHT. More researches can be practiced for further investigation. But the method has several advantages, including rapid analysis, simple mobile phase, and simple sample preparation. It was success- fully used for the analysis of compatibility study of a formulation prepared in our laboratory and suitable for routine analysis in quality-control laboratories.

\section{Acknowledgements}

This research was partially supported by the excellent young scientists funds (No.2006 J23JH024) of the Science and Technology Foundation of Dalian, China.

\section{References}

[1] W. Y. Jiang, "Therapeutic Wisdom in Traditional Chinese Medicine: A Perspective from Modern Science," Trends in Pharmacological Sciences, Vol. 26, No. 11, 2005, pp. 558-563. doi:10.1016/j.tips.2005.09.006

[2] B. T. J. Van, "Chemical Analysis of Ginkgo Biloba
Leaves and Extracts,” Journal of Chromatography A, Vol. 967, No. 1, 2002, pp. 21-55. doi:10.1016/S0021-9673(02)00172-3

[3] R. L. J. Graham, C. Graham, S. M. Clean, T. B. Chen, M. O’Rourke, D. Hirst, D. Theakston and C. Shaw, "Identification and Functional Analysis of a Novel Bradykinin Inhibitory Peptide in the Venoms of New World Crotalinae Pit Vipers," Biochemical and Biophysical Research Communications, Vol. 338, No. 3, 2005, pp. 1587-1592. doi:10.1016/j.bbrc.2005.10.130

[4] T. Wu, R. Abdulla, Y. X. Zhao, Y. Yang, J. Chen and H. A. Aisa, "Simultaneous Quantification of Seven Flavonoids in Flos Gossypii by LC," Chromatographia, Vol. 68, No. 5, 2008, pp. 467-470. doi:10.1365/s10337-008-0723-2

[5] X. C. Ma, X. L. Xin, K. X. Liu, B. J. Zhang, F. Y. Li and D. A. Guo, "Simultaneous Determination of Nine Major Flavonoids in Sophora flavescens by RP-LC," Chromatographia, Vol. 68, No. 5-6, 2008, pp. 471-474. doi:10.1365/s10337-008-0719-y

[6] X. W. Shi, J. L. Qi, Y. B. Wu, Y. Fu, Y. Z. Wang and D. Q. Zhang, "Simultaneous Quantification of Six Sesquiterpene Lactones in Inula Britannica L. by RP-LC,” Chromatographia, Vol. 68, No. 3, 2008, pp. 281-285. doi:10.1365/s10337-008-0698-z

[7] J. Kang, L. Zhou, J.B. Sun, J. Han and D. A. Guo, "Chromatographic Fingerprint Analysis and Characterization of Furocoumarins in the Roots of Angelica Dahurica by HPLC/DAD/ESI-MSN Technique," Journal of Pharmaceutical and Biomedical Analysis, Vol. 47, No. 4-5, 2008, pp. 778-785. doi:10.1016/j.jpba.2008.03.010

[8] J. Su, P. Fu, Y. H. Shen, C. Zhang, M. J. Liang, R. H. Liu, H. L. Li and W. D. Zhang, "Simultaneous Analysis of Flavonoids from Hypericum Japonicum Thunb.ex Murray (Hypericaceae) by HPLC-DAD-ESI/MS," Journal of Pharmaceutical and Biomedical Analysis, Vol. 46, No. 2, 2008, pp. 342-348. doi:10.1016/j.jpba.2007.10.032

[9] W. Li, Y. L. Deng, R. J. Dai, Y. H. Yu, M. K. Saeed, L. 
Li, W. W. Meng and X. S. Zhang, "Chromatographic Fingerprint Analysis of Cephalotaxus Sinensis from Various Sources by High-Performance Liquid Chroma- tography-Diodearray Detection-Electrospray IonizationTandem Mass Spectrometry," Journal of Pharmaceutical and Biomedical Analysis, Vol. 45, No. 1, 2007, pp. 38-46. doi:10.1016/j.jpba.2007.05.027

[10] W. Jin, R. L. Ge, Q. J. Wei, T. Y. Bao, H. M. Shi and P. F. Tu, "Development of High-Performance Liquid Chromatographic Fingerprint for the Quality Control of Rheum Tanguticum Maxim.ex Balf.," Journal of Chromatography A, Vol. 1132, No. 1-2, 2006, pp. 320324. doi:10.1016/j.chroma.2006.08.022

[11] S. H. Zhou, "Thirty Cases of Chronic Cholecystitis Treated by Acupuncture and Oral Adiministration of Da Chai Hu Tang," Journal of Traditional Chinese Medicine, Vol. 28, No. 2, 2008, pp. 173-174. doi:10.1016/S0254-6272(08)60039-4

[12] Y. Fumihiko, I. Akira, K. Yasuhiro, M. Akiyo, I. Hiroshige and K. Kazuo, "Effects of Dai-saiko-to (Da-ChaiHu-Tang) on Plasma Lipids and Atherosclerotic Lesions in Female Heterozygous Heritable Kurosawa and Kusanagi Hyper Cholesterolemic (KHC) Rabbits,” Pharmacological Research, Vol. 50, No. 1-2, 2004, pp. 223230. doi:10.1016/j.phrs.2004.02.003

[13] I. Akira, T. I. Osamu, Y. Fumihiko, M. Bunsho, A. Sakae, K. Yasuhiro, K. Kazuo, M. Akiyo and I. Hiroshige, "Inhibitory Effects of Dai-saiko-to (Da-Chai-Hu-Tang) on the Progression of Atherosclerotic Lesions in Kurosawa and Kusanagi Hyper-Cholesterolemic Rabbits,” Journal of Ethnopharmacology, Vol. 63, No. 3, 1998, pp. 209-218. doi:10.1016/S0378-8741(98)00083-X

[14] Y. Masaki, S. Atsushi, J. Hiroko, Y. Naoki and H. Tadashi, "Glucosyl Hesperidin Prevents Endothelial Dysfunction and Oxidative Stress in Spontaneously Hypertensive rats," Nutrition, Vol. 24, No. 5, 2008, pp. 470-476. doi:10.1016/j.nut.2008.01.010

[15] P. Kannampalli, H. P. Sang, C. K. Kyong, "Hesperidin a Flavanoglycone Protects against $\gamma$-irradiation Induced Hepatocellular Damage and Oxidative Stress in Sprague-Dawley Rats,” European Journal of Pharmacology, Vol. 587, No. 1, 2008, pp. 273-280. doi:10.1016/j.ejphar.2008.03.052

[16] G. H. Xu, D. H. Liu, J. C. Chen, X. Q. Ye, Y. Q. Ma and J. Shi, "Juice Components and Antioxidant Capacity of Citrus Varieties Cultivated in China," Food Chemistry, Vol. 106, No. 2, 2008, pp. 545-551. doi:10.1016/j.foodchem.2007.06.046

[17] P. F. Sebastian, W. Cristina, C. P. Alejandro and M. Mariel, "Synergistic Interaction between Hesperidin, a Natural Flavonoid, and Diazepam," European Journal of Pharmacology, Vol. 512, No. 2-3, 2005, pp. 189-198. doi:10.1016/j.ejphar.2005.02.039

[18] K. Gaganjit, T. Naveen and C. Kanwaljit, "Beneficial Effect of Hesperidin on Lipopolysaccharide-induced Hepatotoxicity,” Toxicology, Vol. 226, No. 2-3, 2006, pp. 152-160. doi:10.1016/j.tox.2006.06.018

[19] L. L. Liu, L. K. Gong, H. Wang, Y. Xiao, X. F. Wu, Y. H.
Zhang, X. Xue, X. M. Qi and J. Ren, "Baicalin Inhibits Macrophage Activation by Lipopolysaccharide and Protects Mice from Endotoxin Shock,” Biochemical Pharmacology, Vol. 75, No. 4, 2008, pp. 914-922. doi:10.1016/j.bcp.2007.10.009

[20] H. Y. Lin, S. C. Shen, C. W. Lin, L. Y. Yang and Y. C. Chen, "Baicalein Inhibition of Hydrogen Peroxide-induced Apoptosis via ROS-dependent Heme Oxygenase 1 Gene Expression," Biochimica et Biophysica Acta, Vol. 1773, No. 7, 2007, pp. 1073-1086. doi:10.1016/j.bbamcr.2007.04.008

[21] K. J. Woo, J. H. Lim, S. Suh, Y. K. Kwon, S. W. Shin, S. C. Kim, Y. H. Choi, J. W. Park and T. K. Kwon, "Differential Inhibitory Effects of Baicalein and Baicalin on LPS-induced Cyclooxygenase-2 Expression through Inhibition of C/EBP $\beta$ DNA-binding Activity,” Immunobiology, Vol. 211, No. 5, 2006, pp. 359-368. doi:10.1016/j.imbio.2006.02.002

[22] H. Mika, O. Takashi, F. Harumi, T. Miho, Y. Shin-ichi, U. Sadaharu, N. Koji, Y. Hisako, T. Kachio and M. Akio, "Difference of Growth-inhibitory Effect of Scutellaria Baicalensis-producing Flavonoid Wogonin among $\mathrm{Hu}-$ man Cancer Cells,” Cancer Letters, Vol. 245, No. 1-2, 2007, pp. 269-274. doi:10.1016/j.canlet.2006.01.011

[23] C. S. Chen, N. J. Chen, L. W. Lin, C. C. Hsieh, G. W. Chen and M. T. Hsieh, "Effects of Scutellariae Radix on Gene Expression in HEK 293 Cells Using cDNA Microarray,” Journal of Ethnopharmacology, Vol. 105, No. 3, 2006, pp. 346-351. doi:10.1016/j.jep.2005.11.012

[24] Y. Hu, Y. Yang, Q. D. You, W. Liu, H. Y. Gu, L. Zhao, K. Zhang, W. Wang, X. T. Wang and Q. L. Guo, "Oroxylin A Induced Apoptosis of Human Hepatocellular Carcinoma Cell Line HepG2 was Involved in Its Antitumor Activity," Biochemical and Biophysical Research Communications, Vol. 351, No. 2, 2006, pp. 521-527. doi:10.1016/j.bbrc.2006.10.064

[25] Y. Ding, L. Zhao, H. Mei, S. L. Zhang, Z. H. Huang, Y. Y. Duan and P. Ye, "Exploration of Emodin to Treat Alpha-naphthylisothiocyanate-induced Cholestatic Hepatitis via Anti-inflammatory Pathway," European Journal of Pharmacology, Vol. 590, No. 1-3, 2008, pp. 377-386. doi:10.1016/j.ejphar.2008.06.044

[26] J. M. Cherng, W. Chiang, J. H. Wang, C. M. Lin, C. Y. Lee, C. M. Shih and L. C. Chiang, "Anthraquinones of Edible Wild Vegetable Cassia Tora Stimulate Proliferation of Human CD4 ${ }^{+} \mathrm{T}$ Lymphocytes and Secretion of Interferon-gamma or Interleukin 10," Food Chemistry, Vol. 107, No. 4, 2008, pp. 1576-1580. doi:10.1016/j.foodchem.2007.10.005

[27] X. Zhou, B. A. Song, L. H. Jin, D. Y. Hu, C. L. Diao, G. F. Xu, Z. H. Zou and S. Yang, "Isolation and Inhibitory Activity against ERK Phosphorylation of Hydroxyanthraquinones from Rhubarb,” Bioorganic \& Medicinal Chemistry Letters, Vol. 16, No. 3, 2006, pp. 563-568. doi:10.1016/j.bmcl.2005.10.047

[28] I. Akira, T. I. Osamu, K. Kazuo, I. Hiroshige, Y. Fumihiko, M. Hiroko, K. Masayoshi, H. Masami, T. Hiroshi and M. Teruhiko, "Evaluation of Rhubarb Using Antioxidative Activity as an Index of Pharmacological Use- 
fulness,” Journal of Ethnopharmacology, Vol. 91, No. 1, 2004, pp. 89-94. doi:10.1016/j.jep.2003.11.021

[29] Y. C. Chen, S. C. Shen, W. R. Lee, F. L. Hsu, H. Y. Lin,

C. H. Ko and S. W. Tseng, "Emodin Induces Apoptosis in Human Promyeloleukemic HL-60 Cells Accompanied by Activation of Caspase 3 Cascade but Independent of Reactive Oxygen Species Production,” Biochemical Pharmacology, Vol. 64, No. 12, 2002, pp. 1713-1724. 